\title{
CHEMICAL TOXICITY AND MICROBIAL CONTAMINATION OF GROUND WATERS NEAR AGRICULTURAL AREAS
}

\author{
D. Nageswara Rao ${ }^{1, *}$, G.V. Krishna Mohan ${ }^{1}$ and P.V.S. Machiraju ${ }^{2}$ \\ ${ }^{1}$ Department of Chemistry, Koneru Lakshmaiah Education Foundation, Guntur-522502, India \\ ${ }^{2}$ Department of Chemistry, Pragati Engineering College (A), Surampalem-533437, A.P., India \\ *E-mail: dr.nageswararao888@gmail.com
}

\begin{abstract}
Water is essential for all living organisms. The addition of Chemicals will become a prime source for contamination of groundwaters near Agricultural activity areas. Keeping in view the hectic agricultural activity in West Godavari District of AP, India, it is proposed to assess the chemical toxicity of waters to suggest the remedial measures to protect the quality of waters to safeguard the health of the public residing in the nearby habitations of Agricultural activity areas. 16 Groundwater samples were collected and characterized for physicochemical parameters viz., $\mathrm{pH}$, EC, TDS, TA, TH, DO, $\mathrm{CO}_{3}{ }^{2-}, \mathrm{HCO}_{3}{ }^{-}, \mathrm{Cl}^{-}, \mathrm{SO}_{4}{ }^{2-}, \mathrm{PO}_{4}{ }^{3-}, \mathrm{NO}_{3}{ }^{-}, \mathrm{Ca}^{2+}, \mathrm{Mg}^{2+}, \mathrm{Na}^{+}, \mathrm{K}^{+}$to evaluate the quality of waters to assess their potentials for application. The waters were characterized for metal ions viz., $\mathrm{Cr}, \mathrm{Mn}, \mathrm{Fe}, \mathrm{Co}, \mathrm{Ni}, \mathrm{Cu}$, $\mathrm{Zn}, \mathrm{Pb}, \mathrm{As}$ and $\mathrm{Hg}$. Some waters are observed with Arsenic concentration while the concentrations of other metals are within the permissible limit. Further, the groundwaters were characterized to assess the microbial contamination and the waters were found to contain pathogenic bacterial species which can cause water-borne diseases. A health survey was carried out and the results revealed the sufferings of the people from health problems which are correlated with the presence of pathogenic bacterial species. The research results revealed that waters are with chemical and microbial contamination and proper treatment is to be done for controlling the toxicity.
\end{abstract}

Keywords: Groundwater, Agriculture, Characterization, Quality, Toxicity.

(C) RASĀYAN. All rights reserved

\section{INTRODUCTION}

Groundwater is consumed by more than $90 \%$ population for drinking and other domestic purposes in India ${ }^{1}$. Khan et al. revealed that nearly one-fifth of all water used in the world is used from groundwater resources ${ }^{2}$. The studies of Karthik et al. revealed that higher TDS are due to the interaction of soil with water ${ }^{3}$. The studies of Ibrahim et al., explained that there are regional differences in water characteristics based on geology and climate ${ }^{4}$. Both urban and rural areas depend on Groundwater as the major source for drinking water $^{5}$. The studies of Singh et al. have revealed that the quality of groundwater changes following variation in the physicochemical and biological environment through which it flows ${ }^{6}$. The studies of Talukder, M.S.U.et al., reported that irrigation with poor quality water reduces soil productivity, changes soil characteristics which ultimately reduces the yield ${ }^{7,8}$.

The studies of K. Boudeffa et al. indicated that water from the downstream has been observed with higher physicochemical parametric values compared to the values of other stations ${ }^{9}$. The research work carried out by Deshpande S.M.et al., ${ }^{10}$ on evaluation of groundwater quality and its suitability for drinking and agriculture showed that with higher concentrations of $\operatorname{TDS}(26.66 \%), \mathrm{EC}(26.66 \%)$, Chloride $(33.33 \%)$, total hardness $(60 \%)$ and magnesium $(86.66 \%)$ are indicators of deterioration in the quality of groundwater as per WHO and BIS standards ${ }^{11}$. The research results of Piper, R.G.et al., indicates the water suitability for success or failure of a fish culture operations ${ }^{12}$. Literature survey ${ }^{13}$ explained that the investigation of groundwater quality is considered commonly for aquaculture as it is free of toxic pollutants and contamination with living organisms. The concentration of chemical constituents will be influenced to a greater extent by geological formations and manmade activities determine the groundwater quality. The higher concentration of metal ions in waters can cause metal toxicity. Excessive utilization of pesticides also becomes a source of groundwater contamination. Pesticides like Calcium arsenate, Lead arsenate,

Rasayan J. Chem., 13(2), 1083-1095(2020)

http://dx.doi.org/10.31788/RJC.2020.1325648 
RASĀYAN J. Chem.

Vol. 13 | No. 2 |1083 - 1095| April - June | 2020

Sodium arsenate and arsenic acid will also become a major source of metal pollution in waters ${ }^{14}$. The research studies carried out by A. M. Shala Abazi et al. indicated the pollution of Stinica river waters due to higher physicochemical parametric levels ${ }^{15}$.

Keeping in view the agricultural scenario in the West Godavari region and aquacultural activity in Krishna district it is proposed to carry out the characterization of groundwater near agriculture and aquaculture activity areas to evaluate the chemical toxicity water and also for assessing it's potentials for various applications.

\section{Materials and Methods}

\section{EXPERIMENTAL}

The sampling locations are identified near the Agricultural activity areas of Tadepalligudem of West Godavari of Andhra Pradesh, India. The samples are collected in polythene bottles and stored for analysis as per the standard procedures ${ }^{16}$. The sample code, location along with Coordinates are summarized in Table -1 .

\begin{tabular}{c|l|c|c}
\multicolumn{2}{c}{ Table-1: Sample Code and Location near Agricultural Areas } \\
\hline \multicolumn{1}{c}{$\begin{array}{c}\text { Sample } \\
\text { Code }\end{array}$} & \multicolumn{1}{c|}{ Sample Location } & \multicolumn{2}{c}{ GPS Co-ordinates } \\
\cline { 3 - 4 } & & Longitude & Latitude \\
\hline TGE-1 & Alampuram, near Kanakadurga hotel & 81.57580 & 16.81099 \\
\hline TGE-2 & Near Duvva main road & 81.62159 & 16.78445 \\
\hline TGE-3 & Near Duvva main road & 81.62160 & 16.78445 \\
\hline TGE-4 & Near Duvva road & 81.63793 & 16.77644 \\
\hline TGW-1 & Pentapaadu near Chinna Sivalayam & 81.52636 & 16.78993 \\
\hline TGW-2 & Pentapaadu opposite Goyanakadegree college & 81.52622 & 16.78965 \\
\hline TGW-3 & Pentapaadu nearGoyanakadegree college & 81.52624 & 16.78976 \\
\hline TGW-4 & Pentapaadu nearJaganjivanaraostatue & 81.52762 & 16.77883 \\
\hline TGN-1 & Pentapaadu near Goyanka degree college & 81.52650 & 16.78997 \\
\hline TGN-2 & Pentapaadu near Goyanaka college & 81.52620 & 16.78966 \\
\hline TGN-3 & Eluru Road near Muthyalamma temple, Muthyalambapuram & 81.49187 & 16.82149 \\
\hline TGN-4 & Eluru Road, near the Market yard & 81.51633 & 16.81687 \\
\hline TGS-1 & Pippara Road & 81.52765 & 16.77912 \\
\hline TGS-2 & Pippara Road, Main road & 81.52753 & 16.77882 \\
\hline TGS-3 & Pippara Road, opposite Srojini rice mill & 81.52757 & 16.77878 \\
\hline TGS-4 & Pippara Road, near Sorijini rice mill & 81.52763 & 16.77882 \\
\hline
\end{tabular}

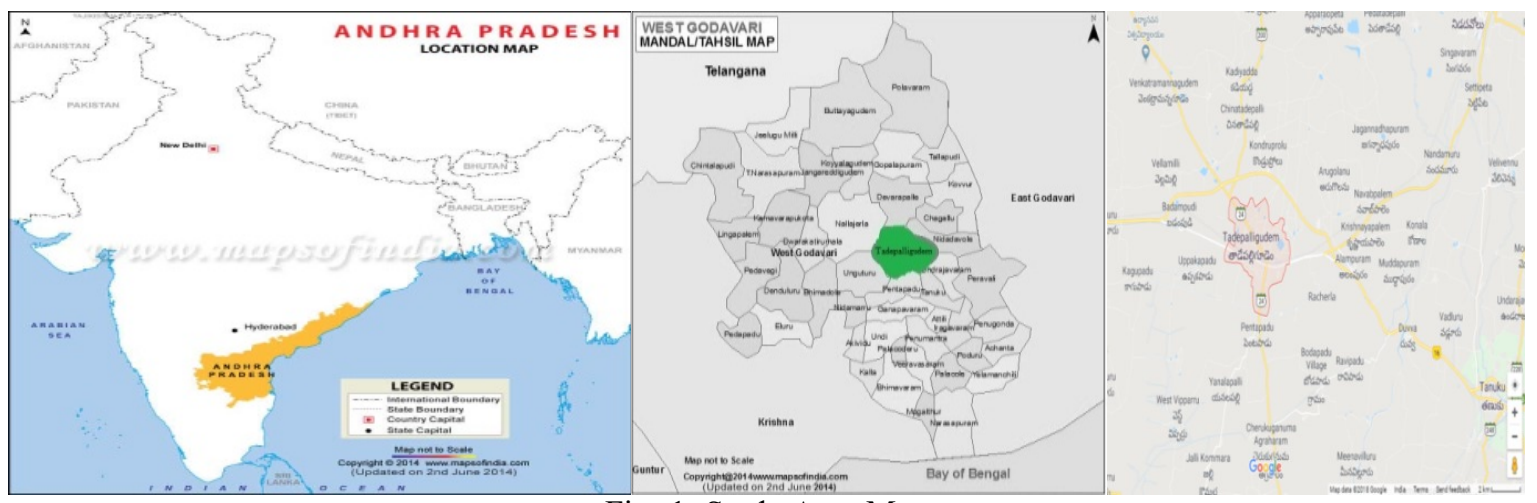

Fig.-1: Study Area Map

The waters were characterized for physicochemical parameters viz., $\mathrm{pH}$, Electrical conductivity (EC), Total Dissolved solids (TDS), Total hardness (TH), Total Alkalinity (TA), Na+, K+, Calcium and Magnesium, Fluoride, Chloride, Sulphate, Nitrate and Phosphate. $\mathrm{pH}$ is determined by $\mathrm{pH}$ Meter (ModelPCS Tester35, Eutech) and Conductivity measured by a portable EC sensor (Electrode based). TDS is determined by TDS Sensor (Electrode based), Total hardness, Total Alkalinity and Chloride are estimated by titrimetry. Sulphate and Phosphate by spectrophotometer (Model-106, Systronics), $\mathrm{Na}^{+}$and $\mathrm{K}^{+}$by Flame photometer (Model-128, Systronics), Nitrate by using Ion-selective electrode (Model-HI 3222 
pH/ORP/ISE Meter, HANNA). The irrigation parametric levels Percent Sodium (\%Na), Sodium Adsorption Ratio (SAR), Residual Sodium Carbonate (RSC), Kelly's ratio (KR), Magnesium Hazard $(\mathrm{MH})$ are determined by the using the relations.

$$
\begin{aligned}
& \text { Percent Sodium }(\% \mathrm{Na})=\frac{\mathrm{Na}^{+} \mathrm{X} 100}{\mathrm{Ca}^{2+}+\mathrm{Mg}^{2+}+\mathrm{Na}^{+}+\mathrm{K}^{+}}(\mathrm{meq} / \mathrm{l}) \\
& \text { Sodium Adsorption Ratio }(\mathrm{SAR})=\frac{\mathrm{Na}^{+}}{\sqrt{\frac{\mathrm{Ca}^{2+}+\mathrm{Mg}^{2+}}{2}}}(\mathrm{meq} / \mathrm{l}) \\
& \text { Residual Sodium Carbonate }(\mathrm{RSC})=\left(\mathrm{CO}_{3}{ }^{2-}+\mathrm{HCO}_{3}^{-}\right)-\left(\mathrm{Ca}^{2+}+\mathrm{Mg}^{2+}\right)(\mathrm{meq} / \mathrm{l}) \\
& \text { Kelly Ratio }(\mathrm{KR})=\frac{\mathrm{Na}^{+}}{\mathrm{Ca}^{2+}+\mathrm{Mg}^{2+}} \\
& \text { Magnesium Hazard }(\mathrm{MH})=\frac{\mathrm{Mg}^{2+}}{\mathrm{Ca}^{2+}+\mathrm{Mg}^{2+}} \mathrm{X} 100
\end{aligned}
$$

Metal ions present in the water sample were characterized by using ICP OES (Avio 200 Perkin ElmerModel). Microbial Analysis of water samples has been carried out by using standard procedure. ${ }^{16-20}$

\section{RESULTS AND DISCUSSION}

pH

$\mathrm{pH}$ levels during pre and post-monsoon seasons range between 7.20-8.55 and 7.72-8.80. the pH of samples TGE-1 during Pre and Post monsoon seasons exceeded the permissible limit and $\mathrm{pH}$ of the remaining samples is within the permissible limits of drinking water standards $6.5-8.5$

\section{Electrical Conductivity}

EC levels range from $703-7600 \mu \mathrm{mhos} / \mathrm{cm}$ and $673-2630 \mu \mathrm{mhos} / \mathrm{cm}$ in groundwaters during pre and postmonsoon seasons. All the EC values of samples except sample TGN-4 exceeded the permissible limit indicating the saline nature of waters. In post-monsoon season EC values except in sample TGN-4 exceeded the permissible limit indicating the saline nature in a TGN-4 water sample.

\section{Total Dissolved Solids}

TDS of groundwater ranges from 499-5360 mg/l during pre-monsoon season while TDS varied from 480$1890 \mathrm{mg} / 1$ in the post-monsoon period and the levels are within the limits of drinking water standards except in sample TGN-4 in both Pre and Post monsoon seasons. Higher TDS values indicate the presence of soluble solids in waters.

\section{Total Hardness}

$\mathrm{TH}$ of groundwaters during pre-monsoon season ranges from 200-2470 mg/l. TH of groundwaters ranges from $300-1400 \mathrm{mg} / \mathrm{l}$ in the post-monsoon season. TH levels in the majority of samples of pre-monsoon and post-monsoon exceeded the permissible limit indicating the hardness of waters.

\section{Total Alkalinity}

TA of waters range from $30-80 \mathrm{mg} / 1$ during pre-monsoon season and are present below the guideline value while in the post-monsoon season it varies from $800-1650 \mathrm{mg} / \mathrm{l}$ and all these levels crossed the permissible limits of drinking water standards. Higher values of TA contribute to change the taste of waters.

$\mathrm{CO}_{3}{ }^{2-} \mathrm{Ion}$

Carbonate levels of waters during pre-monsoon were observed at BDL in all samples except in Two samples TGE-1 and TGS-1 and during post-monsoon season values $180 \mathrm{mg} / 1$ in sample TGE- 1 and $96 \mathrm{mg} / \mathrm{l}$ in TGS-4 and other samples it is at BDL level. 
RASĀYAN J. Chem.

Vol. 13 | No. 2 |1083 - 1095| April - June | 2020

$\mathrm{HCO}_{3}^{-} \mathrm{Ion}$

Bicarbonate values of waters range from $36.6-97.6 \mathrm{mg} / 1$ in both pre-monsoon period and it varies from $780.8-2013.0 \mathrm{mg} / \mathrm{l}$ in the post-monsoon season.

\section{Chloride Ion}

During pre-monsoon season Chloride ion concentration in waters vary from $127.62-3034.52 \mathrm{mg} / \mathrm{l}$ and it ranges from 389.95-2906.90 mg/l during the post-monsoon period. Except in the TGN-4 sample during the pre and post-monsoon period, the Chloride concentrations are within the guideline value of drinking water standards.

\section{Sulphate Ion}

Sulphate in waters of pre-monsoon season varies from $92.86-344.29 \mathrm{mg} / 1$ while it ranges from $60.14-$ $2045.70 \mathrm{mg} / \mathrm{l}$ during the post-monsoon period. In majority waters during pre-monsoon, Sulphate levels are within the permissible limit. During a post-monsoon season in majority waters, the sulphate levels are within the permissible limit.

\section{Nitrate Ion}

In waters, Nitrate ion concentration during pre-monsoon season varies from 4.39-86.9 mg/l and it varies from $4.71-73.8 \mathrm{mg} / 1$ during post-monsoon season. In majority samples during pre and post-monsoon season, Nitrate levels are within the permissible limit.

Table-2: Physicochemical Parametric Values

\begin{tabular}{|c|c|c|c|c|c|c|c|c|c|c|c|c|c|c|c|c|}
\hline \multirow{3}{*}{$\begin{array}{l}\text { Sample } \\
\text { Code }\end{array}$} & \multicolumn{2}{|c|}{ Temp ${ }^{0} \mathrm{C}$} & \multicolumn{2}{|c|}{$\mathrm{pH}$} & \multicolumn{2}{|c|}{$\begin{array}{c}\text { EC } \\
(\mu \mathrm{mhos} / \mathrm{cm})\end{array}$} & \multicolumn{2}{|c|}{$\begin{array}{l}\text { TDS } \\
(\mathrm{mg} / \mathrm{l})\end{array}$} & \multicolumn{2}{|c|}{$\begin{array}{l}\text { Salinity } \\
(\mathrm{mg} / \mathrm{l})\end{array}$} & \multicolumn{2}{|c|}{$\begin{array}{c}\mathrm{TH} \\
(\mathrm{mg} / \mathrm{l})\end{array}$} & \multicolumn{2}{|c|}{$\begin{array}{c}\mathrm{Ca} \\
\text { Hardness } \\
(\mathrm{mg} / \mathrm{l}) \\
\end{array}$} & \multicolumn{2}{|c|}{$\begin{array}{c}\mathrm{Mg} \\
\text { Hardness } \\
(\mathrm{mg} / \mathrm{l})\end{array}$} \\
\hline & \multicolumn{2}{|c|}{ Monsoon } & \multicolumn{2}{|c|}{ Monsoon } & \multicolumn{2}{|c|}{ Monsoon } & \multicolumn{2}{|c|}{ Monsoon } & \multicolumn{2}{|c|}{ Monsoon } & \multicolumn{2}{|c|}{ Monsoon } & \multicolumn{2}{|c|}{ Monsoon } & \multicolumn{2}{|c|}{ Monsoon } \\
\hline & Pre & Post & Pre & Post & Pre & Post & Pre & Post & Pre & Post & Pre & Post & Pre & Post & Pre & Post \\
\hline TGE1 & 29.6 & 30.3 & 8.55 & 8.80 & 1817 & 1630 & 1290 & 1150 & 960 & 858 & 200 & 400 & 150 & 200 & 50 & 200 \\
\hline TGE2 & 29.0 & 30.4 & 7.66 & 8.15 & 2370 & 1520 & 1670 & 1080 & 1260 & 804 & 450 & 300 & 300 & 300 & 150 & BDL \\
\hline TGE3 & 29.5 & 30.2 & 7.85 & 8.07 & 2400 & 1570 & 1700 & 1120 & 1290 & 830 & 390 & 400 & 200 & 300 & 190 & 100 \\
\hline TGE4 & 29.4 & 30.3 & 7.83 & 8.50 & 2140 & 1742 & 1510 & 1240 & 1150 & 922 & 390 & 400 & 350 & 200 & 40 & 200 \\
\hline TGW1 & 29.5 & 30.4 & 8.23 & 8.19 & 3560 & 1600 & 2520 & 1140 & 1940 & 880 & 430 & 500 & 250 & 200 & 180 & 300 \\
\hline TGW2 & 29.4 & 30.3 & 7.92 & 7.92 & 2050 & 1540 & 1450 & 1110 & 1100 & 828 & 380 & 1100 & 100 & 300 & 280 & 800 \\
\hline TGW3 & 29.5 & 30.4 & 7.75 & 7.72 & 4190 & 1650 & 2960 & 1190 & 2300 & 897 & 760 & 600 & 500 & 300 & 260 & 300 \\
\hline TGW4 & 29.5 & 30.4 & 7.71 & 7.80 & 2550 & 1556 & 1800 & 1110 & 1360 & 824 & 500 & 600 & 350 & 300 & 150 & 300 \\
\hline TGN1 & 29.2 & 30.3 & 7.61 & 7.75 & 4200 & 1110 & 2960 & 800 & 2310 & 590 & 520 & 700 & 350 & 300 & 170 & 400 \\
\hline TGN2 & 29.0 & 30.3 & 8.10 & 8.02 & 3800 & 1580 & 2680 & 1130 & 2070 & 841 & 510 & 400 & 300 & 200 & 210 & 200 \\
\hline TGN3 & 29.5 & 30.3 & 7.67 & 7.74 & 5600 & 1510 & 3950 & 1080 & 3140 & 812 & 1490 & 1100 & 1200 & 100 & 290 & 1000 \\
\hline TGN4 & 29.4 & 30.3 & 7.77 & 8.05 & 703 & 673 & 499 & 480 & 359 & 346 & 220 & 300 & 200 & 100 & 20 & 200 \\
\hline TGS1 & 29.4 & 30.2 & 8.49 & 8.04 & 1894 & 2520 & 1340 & 1810 & 1000 & 1380 & 300 & 800 & 250 & 600 & 50 & 200 \\
\hline TGS2 & 29.6 & 30.3 & 7.86 & 8.05 & 1571 & 2630 & 1110 & 1890 & 825 & 1440 & 380 & 600 & 300 & 400 & 80 & 200 \\
\hline TGS3 & 26.6 & 30.2 & 7.20 & 8.03 & 7600 & 2610 & 5360 & 1880 & 4330 & 1430 & 2470 & 1400 & 2200 & 300 & 270 & 1100 \\
\hline TGS4 & 29.5 & 30.3 & 7.22 & 8.48 & 7140 & 1650 & 5060 & 1230 & 4080 & 940 & 2110 & 1400 & 150 & 300 & 1960 & 1100 \\
\hline
\end{tabular}

\section{Phosphate Ion}

In waters, Phosphate ion concentration ranges from $0.37-1.5 \mathrm{mg} / 1$ during the pre-monsoon period while it ranges from 0.01-1.03 mg/l. In post-monsoon season and all the levels are within the permissible limit.

\section{$\mathrm{Ca}^{2+}$ Ion}

Calcium ion concentration in waters of pre and post-monsoon periods vary from 40-880 mg/l and 40-160 $\mathrm{mg} / \mathrm{l}$. In most of the samples during pre and post-monsoon seasons, the $\mathrm{Ca}^{2+}$ levels exceeded the permissible limit. 
RASĀYAN J. Chem.

Vol. 13 | No. 2 |1083 - 1095| April - June | 2020

$\mathrm{Mg}^{2+}$ Ion

Magnesium ion concentration in waters of pre-monsoon season varies from 9.76-478.24 mg/l. Its concentration in post-monsoon samples ranges from $24.4-268.4 \mathrm{mg} / \mathrm{l}$. In most of the samples, $\mathrm{Mg}^{2+}$ concentrations exceeded the permissible limit.

\section{$\mathrm{Na}^{+}$Ion}

Sodium ion concentration in waters in pre and post-monsoon season ranges from 107.4-1909.8 mg/1 and 84.4-710.6 mg/l. In the majority of samples, the values exceeded the guideline value of WHO standards.

\section{$\mathbf{K}^{+}$Ion}

$\mathrm{K}^{+}$ion concentration in waters in pre and post-monsoon season $\mathrm{K}^{+}$ion concentration ranges from 5.6-50.4 $\mathrm{mg} / 1$ and $3.9-158.8 \mathrm{mg} / 1$ respectively. In most samples, $\mathrm{K}^{+}$ion concentration in pre and post-monsoon is within the permissible limit.

Table-3: Physicochemical Parametric Levels

\begin{tabular}{|c|c|c|c|c|c|c|c|c|c|c|c|c|c|c|}
\hline \multirow{3}{*}{$\begin{array}{l}\text { Sample } \\
\text { Code }\end{array}$} & \multicolumn{2}{|c|}{$\begin{array}{c}\text { BiCarbonate } \\
\text { Alkalinity } \\
(\mathrm{mg} / \mathrm{l})\end{array}$} & \multicolumn{2}{|c|}{$\begin{array}{c}\text { Carbonate } \\
\text { Alkalinity } \\
(\mathrm{mg} / \mathrm{l})\end{array}$} & \multicolumn{2}{|c|}{$\begin{array}{l}\text { Hydroxyl } \\
\text { Alkalinity } \\
(\mathrm{mg} / \mathrm{l})\end{array}$} & \multicolumn{2}{|c|}{$\mathrm{TA}(\mathrm{mg} / \mathrm{l})$} & \multicolumn{2}{|c|}{$\mathrm{HCO}_{3}^{-}(\mathrm{mg} / \mathrm{l})$} & \multicolumn{2}{|c|}{$\begin{array}{l}\mathrm{CO}_{3}{ }^{2-} \\
(\mathrm{mg} / \mathrm{l})\end{array}$} & \multicolumn{2}{|c|}{$\begin{array}{c}\mathrm{OH}^{-} \\
(\mathrm{mg} / \mathrm{l})\end{array}$} \\
\hline & \multicolumn{2}{|c|}{ Monsoon } & \multicolumn{2}{|c|}{ Monsoon } & \multicolumn{2}{|c|}{ Monsoon } & \multicolumn{2}{|c|}{ Monsoon } & \multicolumn{2}{|c|}{ Monsoon } & \multicolumn{2}{|c|}{ Monsoon } & \multicolumn{2}{|c|}{ Monsoon } \\
\hline & Pre & Post & Pre & Post & Pre & Post & Pre & Post & Pre & Post & Pre & Post & Pre & Post \\
\hline TGE1 & 50 & 1000 & 20 & 300 & BDL & BDL & 70 & 1300 & 61.0 & 1220 & 12 & 180 & BDL & BDL \\
\hline TGE2 & 70 & 1150 & BDL & BDL & BDL & BDL & 70 & 1150 & 85.4 & 1403 & BDL & BDL & BDL & BDL \\
\hline TGE3 & 70 & 1100 & BDL & BDL & BDL & BDL & 70 & 1100 & & & BDL & BDL & $\mathrm{BDL}$ & BDL \\
\hline TGE4 & 70 & 1430 & BDL & 100 & BDL & BDL & 70 & 1530 & 85.4 & 1744.6 & BDL & 60 & BDL & BDL \\
\hline TGW1 & 80 & 1650 & BDL & BDL & BDL & BDL & 80 & 1650 & 97.6 & 2013 & BDL & BDL & BDL & BDL \\
\hline TGW2 & 60 & 1300 & BDL & BDL & BDL & BDL & 60 & 1300 & 73.2 & 1586 & BDL & BDL & $\mathrm{BDL}$ & BDL \\
\hline TGW3 & 70 & 800 & BDL & BDL & $\mathrm{BDL}$ & BDL & 70 & 800 & 85.4 & 976 & BDL & BDL & BDL & $\mathrm{BDL}$ \\
\hline TGW4 & 50 & 800 & BDL & BDL & $\mathrm{BDL}$ & $\overline{B D L}$ & 50 & 800 & 61.0 & 976 & BDL & BDL & BDL & BDL \\
\hline TGN1 & 80 & 970 & BDL & BDL & BDL & BDL & 80 & 970 & 97.6 & 1183.4 & BDL & BDL & BDL & BDL \\
\hline TGN2 & 80 & 1050 & BDL & BDL & BDL & BDL & 80 & 1050 & 97.6 & 1281 & BDL & BDL & BDL & BDL \\
\hline TGN3 & 50 & 1080 & BDL & BDL & BDL & BDL & 50 & 1080 & 61.0 & 1317.6 & BDL & BDL & BDL & BDL \\
\hline TGN4 & 30 & 1000 & BDL & BDL & BDL & BDL & 30 & 1000 & 36.6 & 1220 & BDL & BDL & BDL & BDL \\
\hline TGS1 & 40 & 870 & 20 & BDL & BDL & BDL & 60 & 870 & 48.8 & 1061.4 & 12 & BDL & BDL & BDL \\
\hline TGS2 & 30 & 1200 & BDL & BDL & $\mathrm{BDL}$ & BDL & 30 & 1200 & 36.6 & 1464 & BDL & BDL & BDL & $\mathrm{BDL}$ \\
\hline TGS3 & 40 & 850 & BDL & BDL & BDL & BDL & 40 & 850 & 48.8 & 1037 & BDL & BDL & BDL & BDL \\
\hline TGS4 & 30 & 640 & BDL & 160 & BDL & BDL & 30 & 800 & 36.6 & 780.8 & BDL & 96 & BDL & BDL \\
\hline
\end{tabular}

Table-4: Physicochemical Parametric Values

\begin{tabular}{|c|c|c|c|c|c|c|c|c|c|c|c|c|c|c|c|c|}
\hline \multirow{3}{*}{$\begin{array}{l}\text { Sample } \\
\text { Code }\end{array}$} & \multicolumn{2}{|c|}{$\begin{array}{c}\mathrm{Ca}^{2+} \\
(\mathrm{mg} / \mathrm{l}) \\
\end{array}$} & \multicolumn{2}{|c|}{$\begin{array}{c}\mathrm{Mg}^{2+} \\
(\mathrm{mg} / \mathrm{l}))\end{array}$} & \multicolumn{2}{|c|}{$\begin{array}{c}\mathrm{Na}^{+} \\
(\mathrm{mg} / \mathrm{l}) \\
\end{array}$} & \multicolumn{2}{|c|}{$\begin{array}{c}\mathrm{K}^{+} \\
(\mathrm{mg} / \mathrm{l}) \\
\end{array}$} & \multicolumn{2}{|c|}{$\begin{array}{c}\mathrm{Cl}^{-} \\
(\mathrm{mg} / \mathrm{l}) \\
\end{array}$} & \multicolumn{2}{|c|}{$\begin{array}{c}\mathrm{SO}_{4}^{2-} \\
(\mathrm{mg} / \mathrm{l}) \\
\end{array}$} & \multicolumn{2}{|c|}{$\begin{array}{l}\mathrm{PO}_{4}{ }^{3-} \\
(\mathrm{mg} / \mathrm{l}) \\
\end{array}$} & \multicolumn{2}{|c|}{$\begin{array}{l}\mathrm{NO}_{3}^{-} \\
(\mathrm{mg} / \mathrm{l})\end{array}$} \\
\hline & \multicolumn{2}{|c|}{ Monsoon } & \multicolumn{2}{|c|}{ Monsoon } & \multicolumn{2}{|c|}{ Monsoon } & \multicolumn{2}{|c|}{ Monsoon } & \multicolumn{2}{|c|}{ Monsoon } & \multicolumn{2}{|c|}{ Monsoon } & \multicolumn{2}{|c|}{ Monsoon } & \multicolumn{2}{|c|}{ Monsoon } \\
\hline & Pre & Post & Pre & Post & Pre & Post & Pre & Post & Pre & Post & Pre & Post & Pre & Post & Pre & Post \\
\hline TGE1 & 60 & 80 & 12.2 & 48.8 & 448.4 & 297.1 & 5.6 & 4.2 & 304.9 & 389.9 & 146.29 & 165.4 & BDL & 0.01 & 56.4 & 67.4 \\
\hline TGE2 & 120 & 120 & 36.6 & BDL & 445.0 & 264.9 & 15.0 & 6.7 & 411.2 & 389.9 & 253.71 & 201.6 & BDL & BDL & 19.4 & 15.1 \\
\hline TGE3 & 80 & 120 & 46.36 & 24.4 & 564.2 & 264.1 & 11.8 & 7.3 & 446.7 & 567.2 & 243.43 & 210.8 & BDL & 0.01 & 26.4 & 15.2 \\
\hline TGE4 & 140 & 80 & 9.76 & 48.8 & 439.6 & 307.9 & 12.0 & 20.9 & 340.3 & 567.2 & 232.00 & 232.4 & BDL & BDL & 16.2 & 19.7 \\
\hline TGW1 & 100 & 80 & 43.92 & 73.2 & 996.0 & 425.0 & 14.0 & 9.7 & 801.2 & 921.7 & 238.71 & 260.3 & BDL & BDL & 32.2 & 27.0 \\
\hline TGW2 & 40 & 120 & 68.32 & 195.2 & 582.4 & 479.3 & 14.8 & 8.7 & 340.3 & 1276.2 & 241.43 & 315.9 & BDL & BDL & 20.5 & 24.1 \\
\hline TGW3 & 200 & 120 & 63.44 & 73.2 & 859.2 & 609.8 & 12.4 & 9.1 & 1120.2 & 1453.4 & 278.57 & 321.8 & BDL & BDL & 34.2 & 53.4 \\
\hline TGW4 & 140 & 120 & 36.6 & 73.2 & 491.8 & 336.3 & 13.2 & 21.2 & 623.9 & 1276.2 & 221.86 & 221.6 & BDL & 0.17 & 17.8 & 40.1 \\
\hline TGN1 & 140 & 120 & & 97.6 & & 272.8 & 9.6 & 10.7 & 942.9 & 1276.2 & 294.71 & 170.1 & BDL & 0.27 & 74.8 & 23.5 \\
\hline TGN2 & 120 & 80 & 51.24 & 48.8 & 882.4 & 159.7 & 11.4 & 158.8 & 872.1 & 1453.4 & 278.14 & 105.3 & BDL & 0.64 & 86.9 & 38.6 \\
\hline TGN3 & 480 & 40 & 70.76 & 244 & 1115.6 & 429.7 & 8.6 & 3.9 & 2041.9 & 2906.9 & 264.00 & 274.8 & BDL & 0.09 & 41.8 & 25.7 \\
\hline TGN4 & 80 & 40 & 44.88 & 48.8 & 107.4 & 84.4 & 14.4 & 7.6 & 127.6 & 1098.9 & 92.86 & 60.1 & 0.37 & 0.21 & 4.4 & 4.71 \\
\hline
\end{tabular}


RASĀYAN J. Chem.

Vol. 13 | No. 2 |1083 - 1095| April - June | 2020

\begin{tabular}{c|c|c|c|c|c|c|c|c|c|c|c|c|c|c|c|c}
\hline TGS1 & 100 & 40 & 12.2 & 48.8 & 390.4 & 465.1 & 9.0 & 4.2 & 340.3 & 1453.5 & 194.71 & 219.0 & 0.43 & 0.21 & 15.6 & 73.8 \\
\hline TGS2 & 120 & 160 & 19.52 & 48.8 & 224.6 & 451.8 & 50.4 & 5.4 & 446.7 & 1630.7 & 122.00 & 236.3 & 0.86 & 0.09 & 11.2 & 48.1 \\
\hline TGS3 & 880 & 120 & 65.88 & 268.4 & 1112.4 & 608.0 & 20.4 & 9.6 & 3034.5 & 2197.9 & 344.29 & 2045.7 & 0.40 & 0.23 & 33.6 & 37.0 \\
\hline TGS4 & 60 & 120 & 478.24 & 268.4 & 1909.8 & 710.6 & 31.2 & 11.8 & 2786.4 & 425.4 & 298.86 & 333.4 & 1.50 & 1.03 & 58.3 & 38.4 \\
\hline
\end{tabular}

Irrigation Parameters

Percent Sodium

Percent Sodium in waters of pre and post-monsoon seasons ranges from 10.1-88.6 me/1 and 46.4-87.8me/1 respectively. Percent $\mathrm{Na}$ exceeded the permissible limit (60me/l) in majority samples

\section{Sodium Adsorption Ratio}

SAR in water samples in pre and post-monsoon seasons range from 1.0-37.3 and 3.0-21.9 me/1. The levels are below the permissible limits of irrigation standards $(26 \mathrm{me} / \mathrm{l})$.

\section{RSC}

RSC values in waters in pre-monsoon season observed at BDL. In post-monsoon season it ranges from 6.2-29.0 me/l and in all samples, it crossed the permissible limit.

\section{Kelly's Ratio}

$\mathrm{KR}$ in water samples in the pre-monsoon period, ranges from 0.13 to $0.62 \mathrm{me} / \mathrm{l}$ and in the post-monsoon period, it ranges from 1.2-7.8 me/l and all the values are above the permissible limit (1.0).

Magnesium Hazard: $\mathrm{MH}$ in waters during pre-monsoon season ranges from 8.9-92.7. During postmonsoon season $\mathrm{MH}$ ranges from $24.5-90.7 \mathrm{me} / 1$ and in majority samples, $\mathrm{MH}$ is within the permissible limit (50) and in the remaining samples, $\mathrm{MH}$ crossed the guideline value indicating the Magnesium hazard.

Table-5: Irrigation Parametric Values of Ground Waters

\begin{tabular}{|c|c|c|c|c|c|c|c|c|c|c|}
\hline \multirow{3}{*}{$\begin{array}{c}\text { Sample } \\
\text { Code }\end{array}$} & \multicolumn{2}{|c|}{$\begin{array}{l}\% \mathrm{Na} \\
(\mathrm{me} / \mathrm{l})\end{array}$} & \multicolumn{2}{|c|}{$\begin{array}{c}\text { SAR } \\
(\mathrm{me} / \mathrm{l})\end{array}$} & \multicolumn{2}{|c|}{$\begin{array}{c}\mathrm{RSC} \\
(\mathrm{me} / \mathrm{l})\end{array}$} & \multicolumn{2}{|c|}{ Kelly Ratio } & \multicolumn{2}{|c|}{$\mathrm{MH}$} \\
\hline & \multicolumn{2}{|c|}{ Monsoon } & \multicolumn{2}{|c|}{ Monsoon } & \multicolumn{2}{|c|}{ Monsoon } & \multicolumn{2}{|c|}{ Monsoon } & \multicolumn{2}{|c|}{ Monsoon } \\
\hline & Pre & Post & Pre & Post & Pre & Post & Pre & Post & Pre & Post \\
\hline TGE1 & 82.6 & 76.1 & 13.8 & 9.2 & BDL & 19.0 & 4.9 & 3.3 & 24.5 & 49.4 \\
\hline TGE2 & 67.5 & 78.4 & 9.2 & 9.4 & BDL & 20.0 & 2.2 & 3.8 & 32.8 & BDL \\
\hline TGE3 & 75.4 & 73.4 & 12.5 & 8.1 & BDL & 18.0 & 3.2 & 2.9 & 48.1 & 24.5 \\
\hline TGE4 & 70.3 & 74.9 & 9.7 & 9.5 & BDL & 25.6 & 2.5 & 3.4 & 10.0 & 49.4 \\
\hline TGW1 & 66.0 & 83.0 & 8.2 & 14.2 & BDL & 29.0 & 2.0 & 5.0 & 41.3 & BDL \\
\hline TGW2 & 52.7 & 76.3 & 5.1 & 11.4 & BDL & 20.0 & 1.3 & 3.3 & 73.2 & 32.8 \\
\hline TGW3 & 75.6 & 86.2 & 17.6 & 18.7 & BDL & 12.0 & 3.2 & 6.6 & 33.7 & 24.5 \\
\hline TGW4 & 88.6 & 87.8 & 37.3 & 21.9 & BDL & 12.0 & 8.4 & 7.8 & 29.5 & 24.5 \\
\hline TGN1 & 80.2 & 78.1 & 19.1 & 11.8 & BDL & 14.5 & 4.2 & 3.7 & 32.2 & 59.4 \\
\hline TGN2 & 70.7 & 80.1 & 11.3 & 13.2 & BDL & 16.0 & 2.5 & 4.2 & 40.6 & 39.4 \\
\hline TGN3 & 55.5 & 81.1 & 9.7 & 15.4 & BDL & 15.7 & 1.3 & 4.5 & 19.1 & 49.4 \\
\hline TGN4 & 81.9 & 69.3 & 14.4 & 8.5 & BDL & 14.1 & 4.9 & 2.5 & 8.9 & 49.4 \\
\hline TGS1 & 87.2 & 62.3 & 24.6 & 6.4 & BDL & 10.5 & 7.1 & 1.7 & 16.3 & 56.5 \\
\hline TGS2 & 83.0 & 46.4 & 19.7 & 4.9 & BDL & 20.0 & 5.1 & 1.8 & 20.7 & 49.4 \\
\hline TGS3 & 49.5 & 63.2 & 9.8 & 8.1 & BDL & 6.2 & 1.0 & 1.7 & 10.7 & 90.7 \\
\hline TGS4 & 10.1 & 53.8 & 1.0 & 3.0 & BDL & 11.4 & 0.1 & 1.2 & 92.7 & 66.1 \\
\hline
\end{tabular}

\section{Correlation Analysis \\ Pre-Monsoon}

EC strongly correlated with TDS, Salinity, Chloride, Sulphate and Sodium.TDS is strongly correlated with TH, Chloride, Sulphate and Sodium. Salinity is strongly correlated with TH, Chloride, Sulphate and Sodium. TH is strongly correlated with Chloride. Chloride is strongly correlated with Sodium. 
RASĀYAN J. Chem.

Vol. 13 | No. 2 |1083 - 1095| April - June | 2020

Correlation data revealed that EC will be caused by the presence of soluble solids which include the salt content of Sodium salts in the form of Chlorides and Sulphates. Salinity is due to the Sodium, Calcium and Magnesium salts in the form of Chlorides and Sulphates. Total Hardness is due to the Chloride salts. The results indicate that the waters are saline in nature and are with hardness making the waters unsuitable for drinking applications.

\section{Post Monsoon}

EC strongly correlated with TDS and Salinity. TDS is strongly correlated with Salinity. Salinity is strongly correlated with $\mathrm{Mg}^{2+}$. Correlation data indicates that EC is due to the presence of Total Dissolved Salts in waters and saline nature of waters. TDS is caused due to the saline nature of waters. TH is due to the Magnesium salts present in waters. The research data indicates that waters are contaminated due to dissolved solids in waters and saline nature of waters and also due to hardness and change the waters unsuitable for drinking and domestic purposes.

\section{Metal Ions}

The concentration of Metal ions viz., $\mathrm{Cr}, \mathrm{Fe}, \mathrm{Co}, \mathrm{Ni}, \mathrm{Cu}, \mathrm{Pb}$ and $\mathrm{Hg}$ in waters of pre and post-monsoon period were found to be nondetectable while Mn concentration is present in pre-monsoon sample TPGE and TPGS at $12.81 \mathrm{ppb}$ and $168.5 \mathrm{ppb}$ Arsenic concentrations in water samples TGE, TGN and TGS Arsenic concentrations are found to be $2.49 \mathrm{ppb}$ of pre-monsoon season $6.391 \mathrm{ppb}$ and 13.02. In sample TGS, Arsenic metal ion exceeded the permissible limit 10ppb of WHO standards. In sample TPG of premonsoon season sample, $\mathrm{Mn}$ ion concentration exceeded the permissible limit of 50ppb of WHO standards. In samples of TGE, TGW, TGN, and TGS of post-monsoon seasons Arsenic concentrations are found to be $10.24 \mathrm{ppb}, 22.3 \mathrm{ppb}, 14.77 \mathrm{ppb}$ and $17.62 \mathrm{ppb}$ respectively. In all waters samples of postmonsoon season, Arsenic metal ion concentration exceeded the permissible limit (10ppb) of WHO standards. Zinc ion concentration in groundwater sample TGS during pre-monsoon sample is $132.2 \mathrm{ppb}$ and is within the permissible of 5000ppb of WHO guideline value while in other samples of pre-monsoon and post-monsoon are found to be not detectable.

Table-6: Correlation Matrix, Pre Monsoon

\begin{tabular}{|c|c|c|c|c|c|c|c|c|c|c|c|c|c|c|c|}
\hline & Temp ${ }^{0} \mathrm{C}$ & $\mathrm{pH}$ & $\begin{array}{c}\mathrm{EC} \\
(\mu \mathrm{mhos} / \mathrm{cm})\end{array}$ & $\begin{array}{c}\text { TDS } \\
(\mathrm{mg} / \mathrm{l})\end{array}$ & $\begin{array}{l}\text { Salinity } \\
(\mathrm{mg} / \mathrm{l})\end{array}$ & $\begin{array}{c}\mathrm{TH} \\
(\mathrm{mg} / \mathrm{l})\end{array}$ & $\begin{array}{c}\mathrm{TA} \\
(\mathrm{mg} / \mathrm{l})\end{array}$ & $\begin{array}{c}\mathrm{Cl}^{-} \\
(\mathrm{mg} / \mathrm{l})\end{array}$ & $\begin{array}{l}\mathrm{SO}_{4}^{2-} \\
(\mathrm{mg} / \mathrm{l})\end{array}$ & $\begin{array}{l}\mathrm{NO}_{3}^{-} \\
(\mathrm{mg} / \mathrm{l})\end{array}$ & $\begin{array}{l}\mathrm{PO}_{4}^{3-} \\
(\mathrm{mg} / \mathrm{l})\end{array}$ & $\begin{array}{c}\mathrm{Na}^{+} \\
(\mathrm{mg} / \mathrm{l})\end{array}$ & $\begin{array}{c}\mathrm{K}^{+} \\
(\mathrm{mg} / \mathrm{l})\end{array}$ & $\begin{array}{c}\mathrm{Ca}^{2+} \\
(\mathrm{mg} / \mathrm{l})\end{array}$ & $\begin{array}{l}\mathrm{Mg}^{2+} \\
(\mathrm{mg} / \mathrm{l})\end{array}$ \\
\hline Temp ${ }^{0} \mathrm{C}$ & 1 & & & & & & & & & & & & & & \\
\hline $\mathrm{pH}$ & 0.466 & 1 & & & & & & & & & & & & & \\
\hline $\mathrm{EC}(\mu \mathrm{mhos} / \mathrm{cm})$ & -0.555 & -0.648 & 1 & & & & & & & & & & & & \\
\hline TDS(mg/l) & -0.553 & -0.648 & 1 & 1 & & & & & & & & & & & \\
\hline Salinity (mg/l) & -0.558 & -0.654 & 1 & 1 & 1 & & & & & & & & & & \\
\hline $\mathrm{TH}(\mathrm{mg} / \mathrm{l})$ & -0.634 & -0.731 & 0.934 & 0.934 & 0.940 & 1 & & & & & & & & & \\
\hline TA (mg/l) & 0.159 & 0.477 & -0.163 & -0.164 & -0.180 & -0.455 & 1 & & & & & & & & \\
\hline $\mathrm{Cl}^{-}(\mathrm{mg} / \mathrm{l})$ & -0.580 & -0.690 & 0.974 & 0.974 & 0.978 & 0.985 & -0.371 & 1 & & & & & & & \\
\hline $\mathrm{SO}_{4}{ }^{2-}(\mathrm{mg} / \mathrm{l})$ & -0.512 & -0.538 & 0.815 & 0.814 & 0.807 & 0.652 & 0.289 & 0.691 & 1 & & & & & & \\
\hline $\mathrm{NO}_{3}{ }^{-}(\mathrm{mg} / \mathrm{l})$ & -0.081 & -0.024 & 0.497 & 0.497 & 0.489 & 0.245 & 0.404 & 0.367 & 0.498 & 1 & & & & & \\
\hline $\mathrm{PO}_{4}{ }^{3-}(\mathrm{mg} / \mathrm{l})$ & -0.038 & -0.385 & 0.337 & 0.339 & 0.351 & 0.491 & -0.740 & 0.464 & -0.057 & -0.023 & 1 & & & & \\
\hline $\mathrm{Na}^{+}(\mathrm{mg} / \mathrm{l})$ & -0.226 & -0.515 & 0.907 & 0.909 & 0.906 & 0.779 & -0.053 & 0.850 & 0.754 & 0.606 & 0.405 & 1 & & & \\
\hline $\mathrm{K}^{+}(\mathrm{mg} / \mathrm{l})$ & -0.041 & -0.359 & 0.097 & 0.098 & 0.105 & 0.244 & -0.647 & 0.210 & -0.181 & -0.221 & 0.741 & 0.074 & 1 & & \\
\hline $\mathrm{Ca}^{2+}(\mathrm{mg} / \mathrm{l})$ & -0.844 & -0.497 & 0.665 & 0.663 & 0.667 & 0.742 & -0.255 & 0.710 & 0.495 & 0.033 & -0.014 & 0.305 & 0.014 & 1 & \\
\hline $\mathrm{Mg}^{2+}(\mathrm{mg} / \mathrm{l})$ & 0.037 & -0.524 & 0.595 & 0.598 & 0.604 & 0.620 & -0.425 & 0.631 & 0.352 & 0.300 & 0.764 & 0.781 & 0.348 & -0.062 & 1 \\
\hline
\end{tabular}

Table-7: Correlation Matrix, Post Monsoon

\begin{tabular}{|c|c|c|c|c|c|c|c|c|c|c|c|c|c|c|c|}
\hline & Temp ${ }^{0} \mathrm{C}$ & $\mathrm{pH}$ & $\begin{array}{c}\mathrm{EC} \\
(\mu \mathrm{mhos} / \mathrm{cm})\end{array}$ & $\begin{array}{c}\mathrm{TDS} \\
(\mathrm{mg} / \mathrm{l})\end{array}$ & $\begin{array}{c}\text { Salinity } \\
(\mathrm{mg} / \mathrm{l})\end{array}$ & $\begin{array}{c}\mathrm{TH} \\
(\mathrm{mg} / \mathrm{l})\end{array}$ & $\begin{array}{c}\mathrm{TA} \\
(\mathrm{mg} / \mathrm{l})\end{array}$ & $\begin{array}{c}\mathrm{Cl}^{-} \\
(\mathrm{mg} / \mathrm{l})\end{array}$ & $\begin{array}{c}\mathrm{SO}_{4}{ }^{2-} \\
(\mathrm{mg} / \mathrm{l})\end{array}$ & $\begin{array}{c}\mathrm{NO}_{3}^{-} \\
(\mathrm{mg} / \mathrm{l})\end{array}$ & $\begin{array}{l}\mathrm{PO}_{4}{ }^{3-} \\
(\mathrm{mg} / \mathrm{l})\end{array}$ & $\begin{array}{l}\mathrm{Na}^{+} \\
(\mathrm{mg} / \mathrm{l})\end{array}$ & $\begin{array}{c}\mathrm{K}^{+} \\
(\mathrm{mg} / \mathrm{l})\end{array}$ & $\begin{array}{c}\mathrm{Ca}^{2+} \\
(\mathrm{mg} / \mathrm{l}) \\
\end{array}$ & $\begin{array}{l}\mathrm{Mg}^{2+} \\
(\mathrm{mg} / \mathrm{l})\end{array}$ \\
\hline Temp ${ }^{0} \mathrm{C}$ & 1 & & & & & & & & & & & & & & \\
\hline $\mathrm{pH}$ & -0.119 & 1 & & & & & & & & & & & & & \\
\hline $\mathrm{EC}(\mu \mathrm{mhos} / \mathrm{cm})$ & -0.396 & 0.087 & 1 & & & & & & & & & & & & \\
\hline
\end{tabular}


RASĀYAN J. Chem.

Vol. 13 | No. 2 |1083 - 1095| April - June | 2020

\begin{tabular}{|c|c|c|c|c|c|c|c|c|c|c|c|c|c|c|c|}
\hline TDS(mg/l) & -0.399 & 0.085 & 0.999 & 1 & & & & & & & & & & & \\
\hline Salinity (mg/l) & -0.391 & 0.086 & 0.998 & 0.999 & 1 & & & & & & & & & & \\
\hline $\mathrm{TH}(\mathrm{mg} / \mathrm{l})$ & -0.341 & -0.151 & 0.371 & 0.396 & 0.405 & 1 & & & & & & & & & \\
\hline TA (mg/l) & 0.186 & 0.436 & -0.078 & -0.097 & -0.093 & -0.386 & 1 & & & & & & & & \\
\hline $\mathrm{Cl}^{-}(\mathrm{mg} / \mathrm{l})$ & -0.197 & -0.666 & 0.286 & 0.285 & 0.287 & 0.467 & -0.297 & 1 & & & & & & & \\
\hline $\mathrm{SO}^{2-}(\mathrm{mg} / \mathrm{l})$ & -0.383 & -0.060 & 0.525 & 0.530 & 0.530 & 0.601 & -0.247 & 0.396 & 1 & & & & & & \\
\hline $\mathrm{NO}_{3}^{-}(\mathrm{mg} / \mathrm{l})$ & -0.128 & 0.181 & 0.607 & 0.606 & 0.608 & 0.163 & -0.264 & 0.105 & 0.066 & 1 & & & & & \\
\hline $\mathrm{PO}_{4}{ }^{3-}(\mathrm{mg} / \mathrm{l})$ & -0.162 & 0.142 & -0.029 & 0.001 & 0.010 & 0.428 & -0.477 & -0.062 & 0.041 & 0.097 & 1 & & & & \\
\hline $\mathrm{Na}^{+}(\mathrm{mg} / \mathrm{l})$ & -0.050 & -0.030 & 0.558 & 0.581 & 0.594 & 0.785 & -0.274 & 0.231 & 0.484 & 0.421 & 0.231 & 1 & & & \\
\hline $\mathrm{K}^{+}(\mathrm{mg} / \mathrm{l})$ & 0.018 & -0.051 & -0.078 & -0.080 & -0.087 & -0.212 & -0.038 & 0.063 & -0.128 & 0.025 & 0.444 & -0.355 & 1 & & \\
\hline $\mathrm{Ca}^{2+}(\mathrm{mg} / \mathrm{l})$ & 0.172 & -0.096 & 0.279 & 0.288 & 0.282 & 0.139 & -0.120 & -0.192 & 0.225 & -0.010 & 0.023 & 0.342 & -0.101 & 1 & \\
\hline $\mathrm{Mg}^{2+}(\mathrm{mg} / \mathrm{l})$ & -0.244 & -0.116 & 0.160 & 0.184 & 0.194 & 0.949 & -0.283 & 0.486 & 0.575 & -0.021 & 0.419 & 0.667 & -0.154 & 0.043 & 1 \\
\hline
\end{tabular}
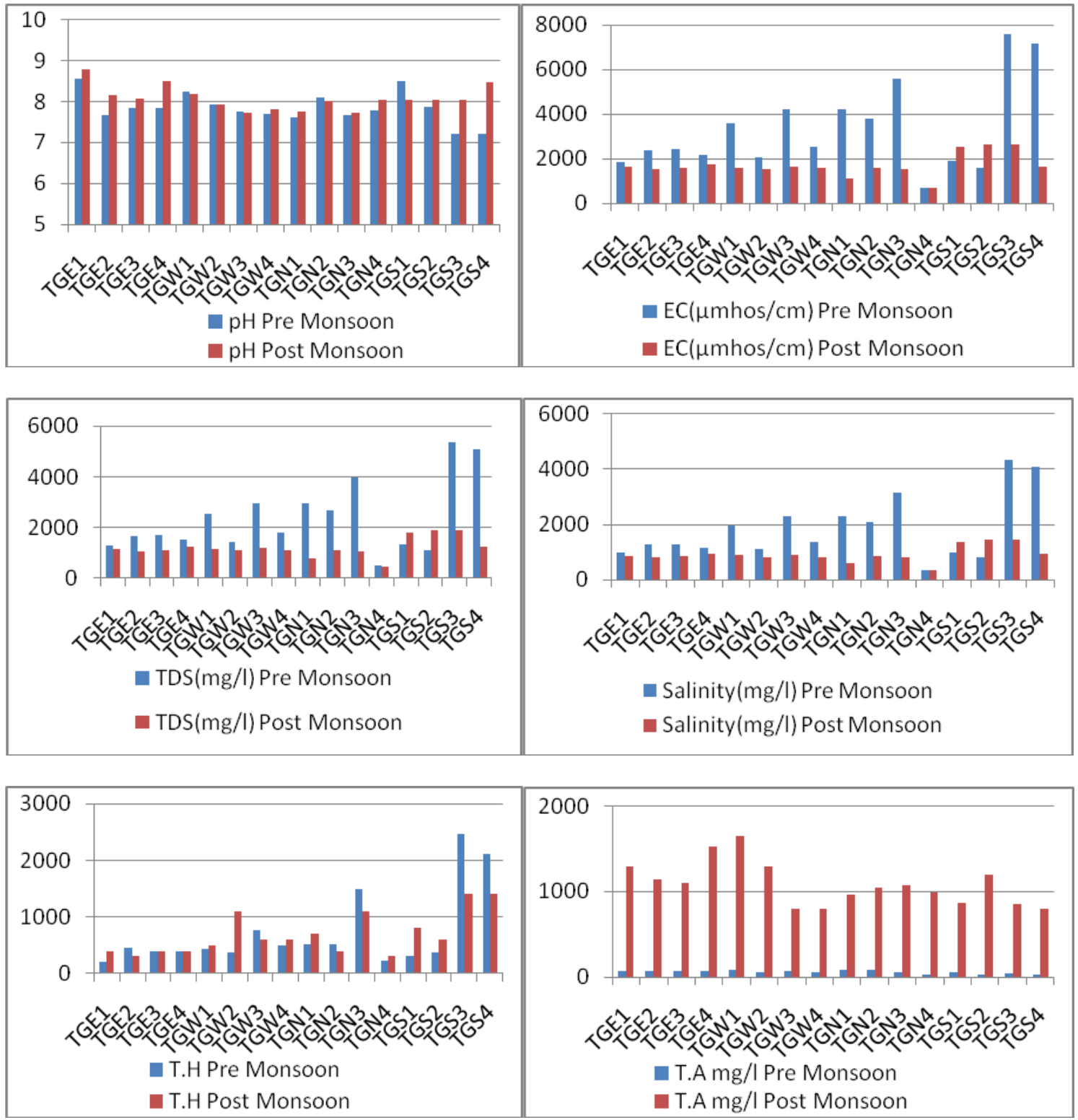
RASĀYAN J. Chem.

Vol. 13 | No. 2 |1083 - 1095| April - June | 2020
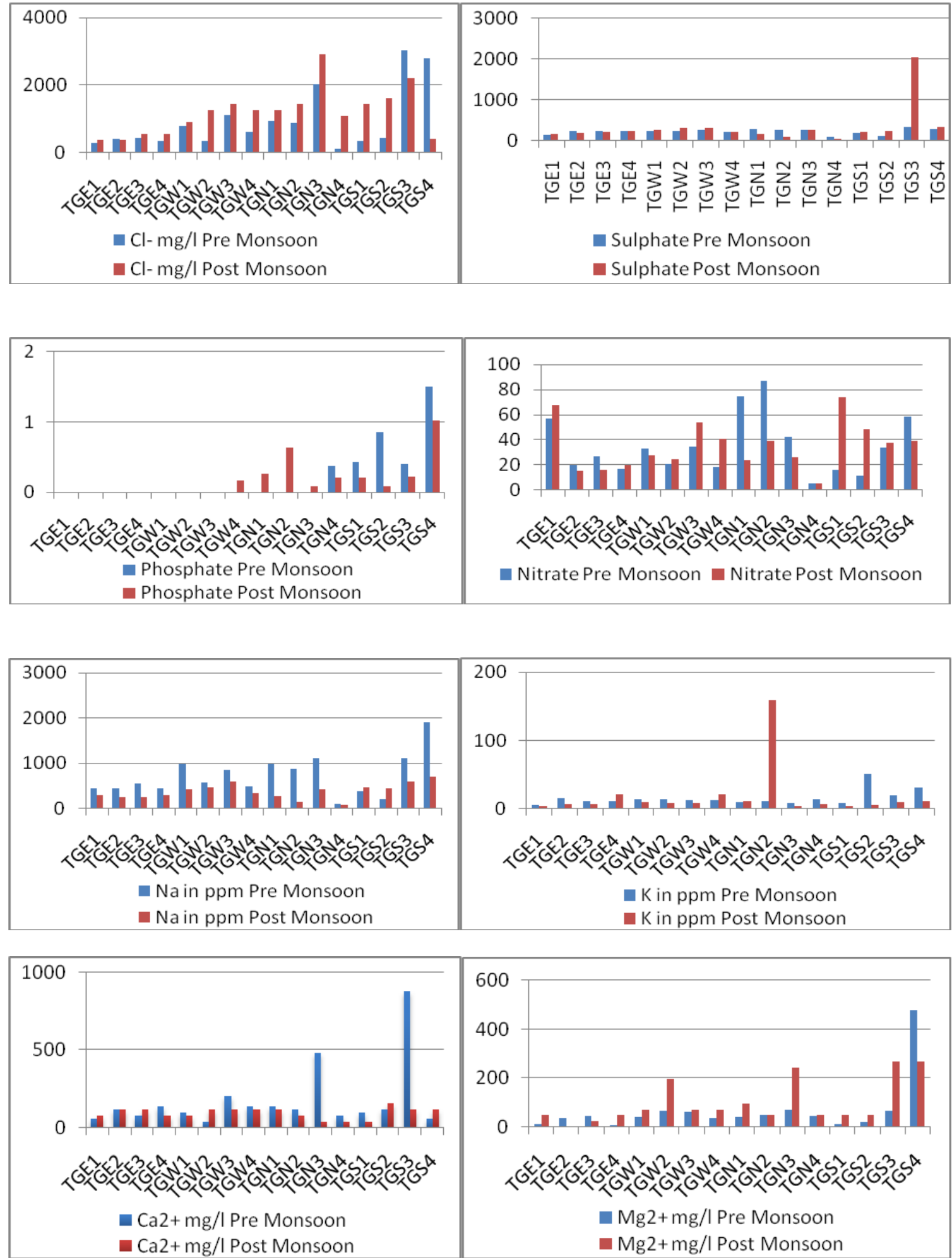
RASĀYAN J. Chem.

Vol. 13 | No. 2 |1083 - 1095| April - June | 2020
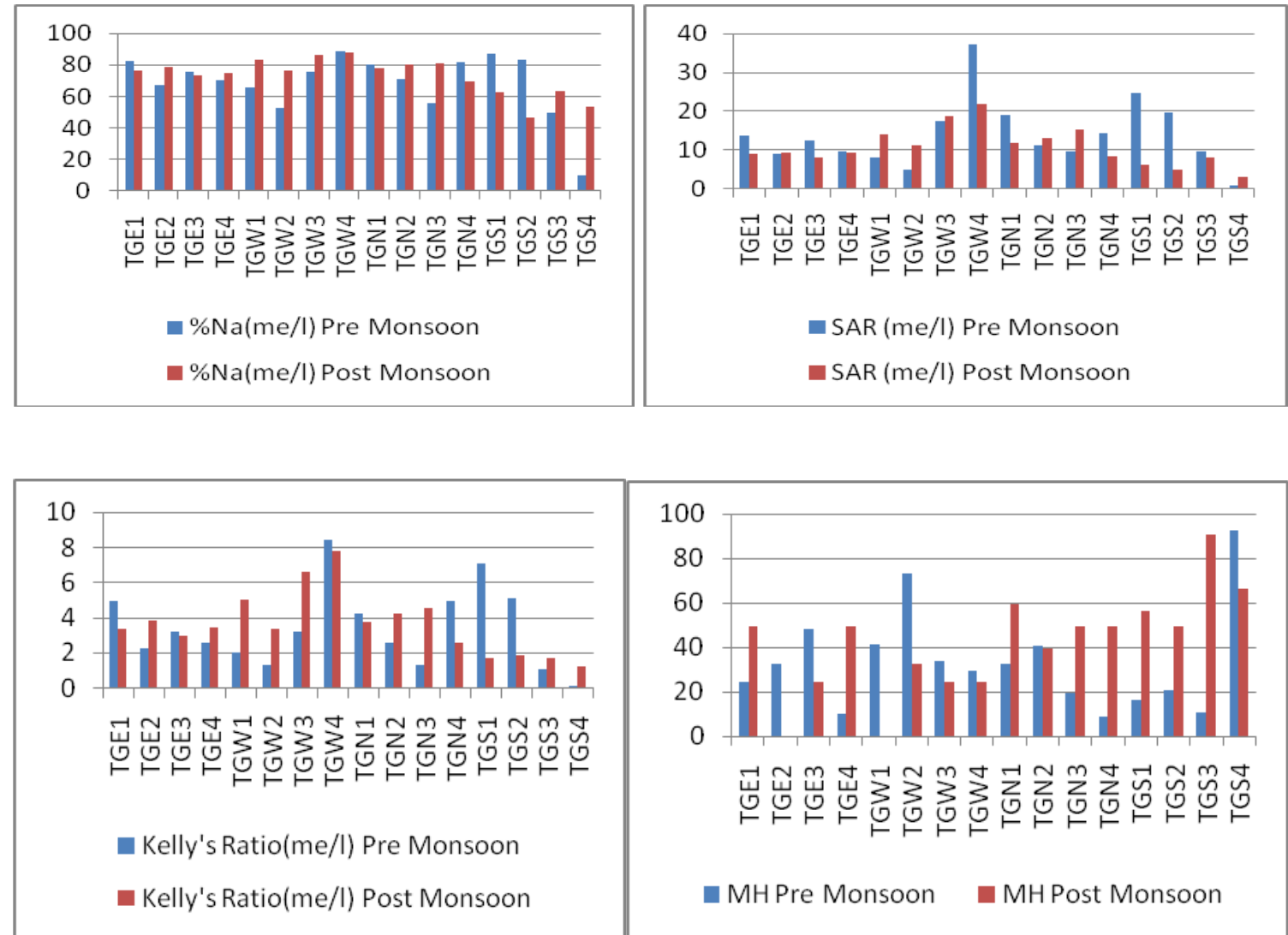

Fig.-2: Graphical Representation of Physicochemical Parametric values

Table-8: Concentration of Metal ions

\begin{tabular}{c|c|c|c|c|c|c|c|c|c|c}
\hline \multirow{3}{*}{ Sample Code } & \multicolumn{2}{|c|}{ Cr (ppb) } & \multicolumn{2}{c|}{ Mn $(\mathrm{ppb})$} & \multicolumn{2}{c|}{ Fe $(\mathrm{ppb})$} & \multicolumn{2}{c|}{ Co $(\mathrm{ppb})$} & \multicolumn{2}{c}{ Ni (ppb) } \\
\cline { 2 - 11 } & \multicolumn{2}{|c|}{ Monsoon } & \multicolumn{2}{c|}{ Monsoon } & \multicolumn{2}{c|}{ Monsoon } & \multicolumn{2}{c|}{ Monsoon } & \multicolumn{2}{c}{ Monsoon } \\
\cline { 2 - 10 } & Pre & Post & Pre & Post & Pre & Post & Pre & Post & Pre & Post \\
\hline TGE & ND & ND & 12.81 & ND & ND & ND & ND & ND & ND & ND \\
\hline TGW & ND & ND & ND & ND & ND & ND & ND & ND & ND & ND \\
\hline TGN & ND & ND & 5.799 & ND & ND & ND & ND & ND & ND & ND \\
\hline TGS & ND & ND & 168.5 & ND & ND & ND & ND & ND & ND & ND \\
\hline
\end{tabular}

Table-9: Concentration of Metal ions

\begin{tabular}{c|c|c|c|c|c|c|c|c|c|c}
\hline \multirow{3}{*}{ Sample Code } & \multicolumn{2}{|c|}{$\mathrm{Cu}(\mathrm{ppb})$} & \multicolumn{2}{c|}{$\mathrm{Zn}(\mathrm{ppb})$} & \multicolumn{2}{c|}{ Pb $(\mathrm{ppb})$} & \multicolumn{2}{c|}{ As (ppb) } & \multicolumn{2}{c}{ Hg (ppb) } \\
\cline { 2 - 11 } & \multicolumn{2}{|c|}{ Monsoon } & \multicolumn{2}{c|}{ Monsoon } & \multicolumn{2}{c|}{ Monsoon } & \multicolumn{2}{c}{ Monsoon } & \multicolumn{2}{c}{ Monsoon } \\
\cline { 2 - 11 } & Pre & Post & Pre & Post & Pre & Post & Pre & Post & Pre & Post \\
\hline TGE & ND & ND & ND & ND & ND & ND & 2.494 & 10.24 & ND & ND \\
\hline TGW & ND & ND & ND & ND & ND & ND & ND & 22.32 & ND & ND \\
\hline TGN & ND & ND & ND & ND & ND & ND & 6.391 & 14.77 & ND & ND \\
\hline TGS & ND & ND & 132.2 & ND & ND & ND & 13.02 & 17.62 & ND & ND \\
\hline
\end{tabular}

Table-10: Details of MPN Index and Bacterial Species identified in Waters

\begin{tabular}{c|c|c|c|c|c|c|c|c|c}
\hline $\begin{array}{c}\text { Sample } \\
\text { Code }\end{array}$ & $\begin{array}{c}\text { MPN- } \\
\text { Count }\end{array}$ & $\begin{array}{c}\text { No. of Bacterial } \\
\text { colonies on } \\
\text { EMB }\end{array}$ & $\begin{array}{c}\text { BORPHOLOGY ON } \\
\text { EMMB }\end{array}$ & $\begin{array}{c}\text { Gram's } \\
\text { Nature }\end{array}$ & I ( IMViC TESTS & MR & VP & C & Bacteria Species \\
\hline TGE-1 & 5 & 1 & Colourless & $-\mathrm{ve}$ & + & + & - & + & CitrobacterFreundii \\
\hline TGE-2 & 11 & 1 & Colourless & $-\mathrm{ve}$ & - & - & - & - & Pseudomonas \\
\hline
\end{tabular}


RASĀYAN J. Chem.

Vol. 13 | No. 2 |1083 - 1095| April - June | 2020

\begin{tabular}{c|c|c|c|c|c|c|c|c|c}
\hline TGW-1 & 9 & 1 & Colourless & $-\mathrm{ve}$ & + & + & - & + & Citrobacter \\
\hline TGW-2 & 4 & 2 & Colourless & $-\mathrm{ve}$ & + & + & - & + & Citrobacter \\
\hline TGN-1 & 5 & 1 & Colourless & $-\mathrm{ve}$ & - & - & - & - & Pseudomonas \\
\hline TGN-2 & 14 & 1 & Colourless & $-\mathrm{ve}$ & - & - & - & - & Pseudomonas \\
\hline TGS-1 & 25 & 2 & Colourless & $-\mathrm{ve}$ & + & + & - & + & Citrobacter \\
\hline TGS-2 & 32 & 2 & Colourless & $-\mathrm{ve}$ & - & - & - & - & Pseudomonas \\
\hline
\end{tabular}

*I $=$ Indole, $\mathrm{MR}=$ MethylRed, $\mathrm{VP}=$ VogesProskauer, $\mathrm{C}=$ Citarte

\section{Microbial Analysis}

The water sample TGE-1 was found with MPN count of 5 and bacterial species like CitrobacterFreundii. Water sample TGE-2 is observed with MPN count 11 and the bacterial species identified is Pseudomonas. Water sample TGW-1 is having MPN count 9 and Citrobacter bacterial species were identified. Water sample TGW-2 is observed with MPN count 4 and the bacterial species present is Citrobacter. Water sample TGN-1 is observed with MPN count 5 and the bacterial species identified is Pseudomonas. Water sample TGN-2 is observed with MPN count 14 and the bacterial species identified is Pseudomonas. Water sample TGS-1 is observed with MPN count 25 and the bacterial species identified is Citrobacter. Water sample TGS-2 is observed with MPN count 32 and the bacterial species identified is Pseudomonas. Health Survey: The health survey results indicated the sufferings of children, youth, middle-aged people and senior citizens of 60 years and above with various health problems like fever, gastrointestinal troubles, typhoid and skin diseases.

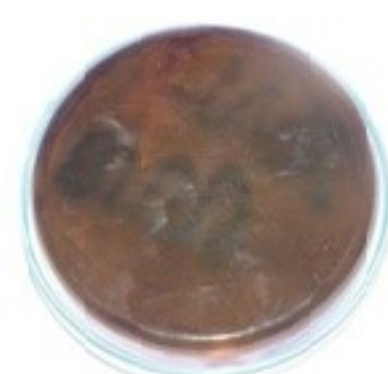

TGE-1: Citrobacter

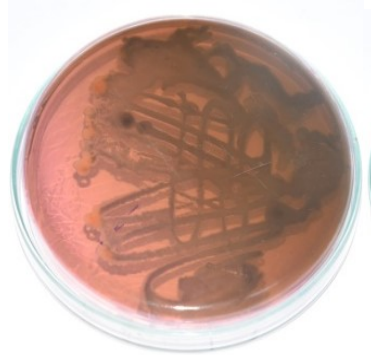

TGN-1: Pseudomonas

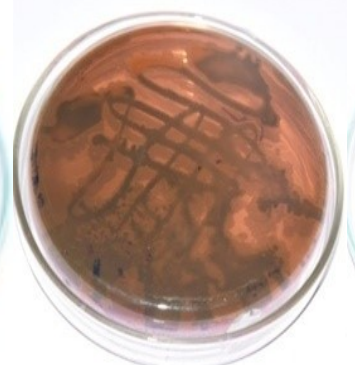

TGE-2: Pseudomonas

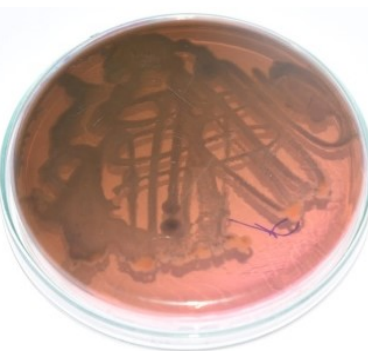

TGN-2: Pseudomonas

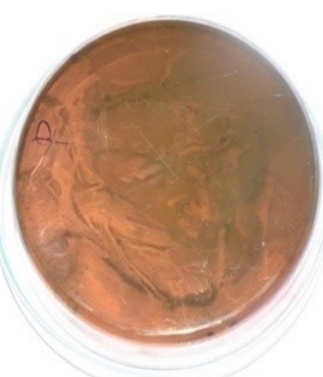

TGW-1: Citrobacter

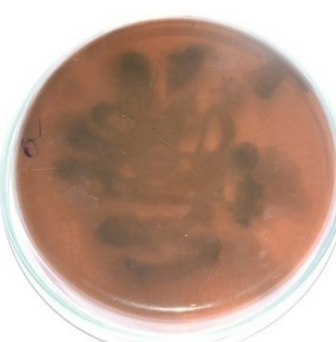

TGS-1: Citrobacter

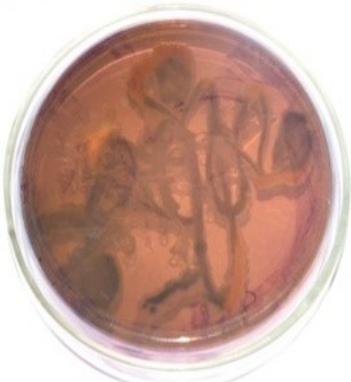

TGW-2: Citrobacter

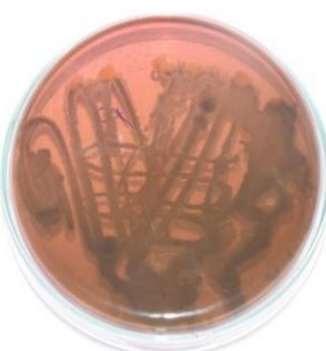

TGS-2: Pseudomonas

Fig.-3: Photographs of Identified Bacterial species in the Waters

Table-11: Health Survey of Public and Disease experienced.

\begin{tabular}{c|c|c|c|c|c|c}
\hline \multirow{2}{*}{$\begin{array}{c}\text { Age in } \\
\text { Yrs }\end{array}$} & Fever & $\begin{array}{c}\text { Gastro } \\
\text { Intestinal } \\
\text { Troubles }\end{array}$ & $\begin{array}{c}\text { Joint } \\
\text { Pains }\end{array}$ & Typhoid & $\begin{array}{c}\text { Skin } \\
\text { Allergy }\end{array}$ & \multirow{2}{*}{ Total } \\
\hline $1-10$ & 3 & 4 & 2 & 2 & 1 & 12 \\
\hline $11-20$ & 4 & 2 & 0 & 2 & 0 & 8 \\
\hline $21-30$ & 1 & 5 & 1 & 0 & 0 & 7 \\
\hline $31-40$ & 1 & 1 & 0 & 0 & 0 & 2 \\
\hline $41-50$ & 5 & 8 & 0 & 0 & 2 & 15 \\
\hline
\end{tabular}


RASĀYAN J. Chem.

Vol. 13 | No. 2 |1083 - 1095| April - June | 2020

\begin{tabular}{c|c|c|c|c|c|c}
\hline $51-60$ & 4 & 5 & 0 & 1 & 1 & 11 \\
\hline Above 60 & 1 & 3 & 1 & 1 & 0 & 6 \\
\hline Total & 19 & 28 & 4 & 6 & 4 & 61 \\
\hline
\end{tabular}

$\mathrm{NE}=$ Not Expressed

\section{CONCLUSION}

The $\mathrm{pH}$ of ground waters is mainly within the permissible limits. Higher EC of waters revealed the saline nature. Higher TDS in waters confirm the soluble solid matter in waters which can deplete the quality of waters. The higher salinity of waters can increase the EC values and can cause saline nature to waters. Higher $\mathrm{TH}$ values in all most all waters indicate the encrustation nature of waters indicating their unsuitability for domestic purposes. TA of waters during pre-monsoon season is within the permissible limits while TA of waters during post-monsoon season exceeded the permissible limits and change the taste of waters and make the waters unfit for drinking purposes. Higher values of chloride in waters of both seasons can cause corrosive nature to waters. Higher values of Sulphate in fifty percent of waters reveal that in ground waters of such locations are having interactions with some effluent waters discharging from the surrounding areas. Nitrate levels in majority samples are below the permissible limits and only in some samples the levels are higher due to discharge of agricultural run-off into the groundwater sources. Phosphate levels are within the permissible limits indicating the non-discharge of agricultural run-off containing the phosphatic fertilizer residues into groundwater sources. Calcium ion concentrations in majority samples of both pre and post-monsoon seasons crossed the permissible limits indicating their encrustation nature and change the suitability of waters for domestic purposes. Magnesium ion concentrations in the majority of waters of both seasons crossed the permissible limits confirming the magnesium hazard. Sodium-ion concentration in waters of both seasons crossed the guideline value of WHO standards indicating the saline nature of waters and Potassium ion concentrations in majority samples of both seasons are within the permissible limits of WHO standards. The concentration of metal ions $\mathrm{Cr}, \mathrm{Mn}, \mathrm{Fe}, \mathrm{Co}, \mathrm{Ni}, \mathrm{Cu}, \mathrm{Zn}, \mathrm{Pb}$ and $\mathrm{Hg}$ are within the permissible limits. In waters of post-monsoon season, Arsenic metal ion concentration exceeded the permissible limit of $10 \mathrm{ppb}$ of WHO standards indicating the Arsenic toxicity of waters which can cause health effects on the cardiovascular system particularly in children and also cause cancer.

Percent Sodium values of majority samples of both the seasons crossed the permissible limits of irrigation standards $(60 \mathrm{me} / \mathrm{l})$ while SAR levels are within the permissible limits $(26 \mathrm{me} / \mathrm{l})$. Though RSC values of Pre-monsoon season are at Below Detectable Limit, they crossed the permissible limits in waters of postmonsoon season. Higher values of Kelly's ratio revealed the unsuitability of waters for irrigation purposes. Magnesium hazard in the case of majority samples is within the permissible limit (50). Higher $\mathrm{MH}$ values in the remaining samples deplete the quality of groundwaters and reduce the crop yields if used for irrigation purposes.

All water samples were found to contain MPN count as well as the pathogenic bacterial species like Pseudomonas, Citrobacter which can cause water-borne diseases like typhoid, viral fevers and dysentery, etc., A higher concentration of Calcium can also cause health problems like joint pains and bone disorders. The health survey results also revealed that the people who are consuming the water for drinking purposes have been suffering from health problems like typhoid, skin diseases, viral fever and joint pains. These health problems indicate the correlation between the presence of pathogenic bacterial species and the higher concentrations of Calcium in waters. The research revealed that the waters are chemically contaminated with a higher concentration of TDS, EC, Salinity and Total Alkalinity with Arsenic metal toxicity. Further the waters are with microbial contamination and as a result, the health problems will be caused. The waters are to be treated with the available ultra and nano filtration to remove the TDS from waters to minimize the chemical contamination whereas the available activated charcoal adsorption is some other green technologies to be used for the removal of Arsenic toxicity. The existing disinfection and sterilization are used to remove microbial contamination.

\section{ACKNOWLEDGMENT}

The authors express their thanks to the Management of Pragati Engineering College (Autonomous), Surampalem, A. P India for their cooperation for completing the chemical analysis in the R\&D Division. 
RASĀYAN J. Chem.

Vol. 13 | No. 2 |1083 - 1095| April - June | 2020

The authors also convey their thanks to the faculty, Department of Microbiology, Y.N.College (A), Narasapuram for their cooperation in carrying out the microbial analysis.

\section{REFERENCES}

1. C. Ramachandraiah, Right to drinking water in India, Centre for Economic and Social Studies, 56(2004)

2. L.R. Khan and B.C. Basak, Bangladesh Journal of Agriculture, 11(4), 21(1986)

3. K. Karthik, R. Mayildurai, R. Mahalakshmi and S. Karthikeyan, Rasayan Journal of Chemistry, 12(2), 409(2019), DOI:10.31788/RJC.2019.1225005

4. A.K. Ibrahim, A. Usman and A.H. Umar, Journal of Environment, Technology and Sustainable Agriculture, 1(1), 72(2011a)

5. D. P. Gupta, Sunita and J.P. Saharan, Researcher, 1(2), 1(2009)

6. S. Mandeep, S. Kaur and S.S. Sooch, Journal of the Institution of Public Health Engineers, 2, 29(2003)

7. M.S.U. Talukder, S.M. Shirazi and U.K. Paul, Journal of Progressive Agriculturist, 9, 107(1998)

8. S.A. Ibrahim, D. D. Bashir, and A.U. Dikko, Journal of Agriculture and Environment, 1(1), 120(2000)

9. K. Boudeffa, F. Fekrache and N. Bouchareb, Rasayan Journal of Chemistry, 13(1), 168(2020), DOI: $10.31788 /$ RJC.2020.1315255

10. S.M. Deshpande and K.R. Aher, Research Journal of Chemical Sciences, 2(1), 25(2012)

11. R.G. Piper, I.B. McElwain, L. E. Ome, J. P. Mccraren, L.G.Flower and J.R. Leonard, Fish Hatechery Management. U. S. Fish and Wild Life Service, Washington, D.C, (1982)

12. S. A. Arabi, I. I. Funtua, S. A. Alagbe., P. Zabosrki and B.B.M. Dewu, Journal of American Science, 6(12), 664(2010), DOI:10.7537/marsjas121216.01

13. WHO, World Health Organization, Meeting the MDG Drinking Water and Sanitation Target: The Urban and Rural Challenge of the Decade, WHO Library Cataloguing-in-Publication Data (2008).

14. A. Rasool, T. Xiao, A. Farooqi , M. Shafeeque, Y. Liu, M. A. Kamran, I. A. Katsoyiannis and S. A. M. A. S. Eqani, Environmental Geochemistry and Health,39, 847(2017), DOI:10.1007/s10653-016-98558.

15. A. M. Shala Abazi, B. H. Durmishi, F. S. Sallaku, H. S. Çadraku, O. B. Fetoshi, P. H. Ymeri and P. S. Bytyçi, Rasayan Journal of Chemistry, 13(1), 146(2020), DOI:10.31788/RJC.2020.1315344

16. D.S. Ramteke, C. A. Moghe, Manual on Water and Waste Water Analysis National Environmental Engineering Research Institute, Nagpur, India, (1988).

17. APHA Standard Method for the Examination of Water and Waste Water $19^{\text {th }}$ ed., American Public Health Association, Washington, (1992)

18. K. Obiri. Danso and K. Jones, Journal of Applied Microbiology, 86, 603(1999a), DOI:10.1046/j.13652672.1999.00703.x

19. K. Obiri. Danso and K. Jones, Journal of Applied Microbiology, 87,822(1999b), DOI:10.1046/j.13652672.1999.00924.x

20. S. Smruti and I. Sanjeeda, Research Journal of Recent Sciences, 1, 323 (2012)

[RJC-5648/2019] 